\title{
Rebound Intracranial Hypertension: A Complication of Epidural Blood Patching for Intracranial Hypotension
}

P.G. Kranz, T.J. Amrhein, and L. Gray

\begin{abstract}
SUMMARY: Rebound intracranial hypertension is a complication of epidural blood patching for treatment of intracranial hypotension characterized by increased intracranial pressure, resulting in potentially severe headache, nausea, and vomiting. Because the symptoms of rebound intracranial hypertension may bear some similarity to those of intracranial hypotension and literature reports of rebound intracranial hypertension are limited, it may be mistaken for refractory intracranial hypotension, leading to inappropriate management. This clinical report of 9 patients with confirmed rebound intracranial hypertension reviews the clinical characteristics of patients with this condition, emphasizing factors that can be helpful in discriminating rebound intracranial hypertension from refractory spontaneous intracranial hypotension, and discusses treatment.
\end{abstract}

ABBREVIATIONS: $\mathrm{SIH}=$ spontaneous intracranial hypotension; RIH = rebound intracranial hypertension; EBP = epidural blood patching; $\mathrm{LP}=$ lumbar puncture

$E^{\mathrm{p}}$ pidural blood patching (EBP) is considered by many authors to be the treatment of choice in cases of spontaneous intracranial hypotension $(\mathrm{SIH}) .{ }^{1-3}$ Prior investigations have predominantly focused on the diagnostic evaluation of spontaneous intracranial hypotension, optimal methods for localizing the CSF leak, and various approaches to performing epidural blood patching. Relatively less attention has been paid, however, to the postprocedural care of patients with intracranial hypotension, including recognition and management of posttreatment complications.

Rebound intracranial hypertension (RIH) is a potential complication of epidural blood patching characterized by a postprocedural elevation of CSF pressure. ${ }^{4}$ Although RIH has been reported previously, reports are limited to only a few patients. The mechanism underlying this phenomenon remains unknown and the onset of symptoms has been reported over a wide timeframe, ranging from days to years after EBP. Despite the small number of reported cases in the literature, our experience has been that this phenomenon is not uncommon. It is likely, in our opinion, that RIH often goes unrecognized.

The primary clinical manifestation of RIH is headache, which may be accompanied by nausea, emesis, and blurred vision. Be-

Received October 14, 2013; accepted after revision November 10

From the Department of Radiology (P.G.K., L.G.), Duke University Medical Center, Durham, North Carolina; and Department Radiology and Radiological Science (T.J.A.), Medical University of South Carolina, Charleston, South Carolina.

Please address correspondence to Peter G. Kranz, MD, Department of Radiology, Box 3808, Duke University Medical Center, Durham, NC 27710; e-mail: peter.kranz@duke.edu

http://dx.doi.org/10.3174/ajnr.A3841 cause patients with SIH typically also have headache as their primary complaint, and because experience with treating these patients is limited at many centers, the headaches associated with RIH might be mistakenly attributed to refractory SIH. As a consequence, treatment aimed at increasing CSF pressure, such as repeat EBP, will be unhelpful for patients with RIH and could even exacerbate the condition. Awareness of this complication is therefore important.

The purpose of this case series is to describe the presentation of a series of patients with confirmed RIH, with an emphasis on common features that may suggest the diagnosis, as well as potential treatment strategies.

\section{MATERIALS AND METHODS}

\section{Case Series}

This investigation is a retrospective case series of patients with confirmed RIH after EBP. The investigation was approved by our local institutional review board and is Health Insurance Portability and Accountability Act-compliant. A waiver of informed consent was obtained for this retrospective review.

Cases were identified from the investigators' cohort of patients previously treated for intracranial hypotension. Inclusion criteria were history of intracranial hypotension confirmed by a combination of clinical symptoms, brain imaging, and/or decreased CSF pressure; treatment with epidural blood and/or fibrin glue patching with subsequent development of new complaints clinically suspected to be caused by increased intracranial pressure; and repeat lumbar puncture (LP) with a documented opening pressure of $>20 \mathrm{~cm} \mathrm{H}_{2} \mathrm{O}$. Patients with clinically suspected RIH with- 
Pre- and post-EBP characteristics of patients with RIH

\begin{tabular}{|c|c|c|c|c|c|c|c|c|c|c|}
\hline \multirow[b]{2}{*}{ Patient } & \multicolumn{2}{|c|}{$\begin{array}{c}\text { Opening Pressure } \\
\left(\mathrm{cm} \mathrm{H} \mathrm{H}_{2} \mathrm{O}\right)\end{array}$} & \multirow{2}{*}{$\begin{array}{l}\text { Time from EBP } \\
\text { to Repeat LP }\end{array}$} & \multicolumn{2}{|c|}{ Headache Location } & \multirow{2}{*}{$\begin{array}{l}\text { Post-EBP } \\
\text { N/V }{ }^{\mathbf{a}}\end{array}$} & \multirow{2}{*}{$\begin{array}{l}\text { Post-EBP } \\
\text { Blurred Vision }\end{array}$} & \multirow[b]{2}{*}{ Patching Agent } & \multirow{2}{*}{$\begin{array}{l}\text { Patch } \\
\text { Volume }\end{array}$} & \multirow{2}{*}{$\begin{array}{c}\text { Duration of } \\
\text { Acetazolamide } \\
\text { Treatment }\end{array}$} \\
\hline & Pre-EBP & Post-EBP & & Pre-EBP & Post-EBP & & & & & \\
\hline 1 & 6.8 & 25.5 & 6 hours & Occipital & Frontal & Yes & Yes & Fibrin glue & $6 \mathrm{~mL}$ & 5 days \\
\hline 2 & N/A & 28.0 & 3 weeks & Retro-orbital & Peri-orbital & No & No & Blood & $18 \mathrm{~mL}$ & $-{ }^{b}$ \\
\hline 3 & 12.3 & 31.0 & 2 hours & Retro-orbital & Retro-orbital & Yes & No & Blood + fibrin glue & $16 \mathrm{~mL}$ & 5 days \\
\hline 4 & 7.8 & 31.0 & 24 hours & Occipital & Holocephalic & Yes & Yes & Blood & $18 \mathrm{~mL}$ & 6 weeks \\
\hline 5 & 1.0 & $>55.0$ & 2 hours & Holocephalic & Holocephalic & Yes & Yes & Blood & $6 \mathrm{~mL}$ & 2 weeks \\
\hline 6 & 15.0 & 32.0 & 7 days & Occipital & Frontal & Yes & Yes & Blood & $5 \mathrm{~mL}$ & $3+$ years \\
\hline 7 & 10.8 & 24.2 & 24 hours & Occipital & Frontal and peri-orbital & Yes & Yes & Blood & $18 \mathrm{~mL}$ & 4 weeks \\
\hline 8 & 7.0 & 22.0 & 48 hours & Occipital & Frontal & Yes & Yes & Blood & $20 \mathrm{~mL}$ & 6 weeks \\
\hline 9 & 8.0 & 22.0 & 12 months & Occipital & Occipital & Yes & Yes & Blood & $42 \mathrm{~mL}$ & 6 months \\
\hline
\end{tabular}

Note:- N/A indicates data not available.

${ }^{a}$ Nausea/vomiting.

beceived follow-up care at another institution.

out a confirmatory opening pressure measurement were not included.

Pretreatment (ie, before EBP) and post-EBP clinical symptoms, CSF pressure measurements, and treatment details were recorded. In all cases, LP was performed by use of imaging (fluoroscopic or CT fluoroscopic) guidance, and opening pressures were measured with the patient in the lateral decubitus position, with legs extended, resting quietly without talking.

A total of 9 cases were identified that met inclusion criteria. These patients included 6 men and 3 women, with an average age of 44 years (range, 21-61). One patient (patient 8) received an epidural blood patch for post-LP headache after work-up of nonpositional migraine headaches. The remaining 8 patients were treated for SIH. Pre-EBP and post-EBP patient characteristics are summarized in the Table.

\section{Characteristics before Blood Patching}

Pre-EBP MR imaging was available in 7 of 8 patients with SIH; 1 of these pretreatment MRIs was performed without contrast. No MR imaging was performed before EBP in the patient with post-LP headache. Among the patients with pretreatment brain MR imaging, diffuse dural enhancement was present in 3 of 6 (50\%), brain sagging in 5 of 7 (71\%), cerebellar tonsillar ectopia in 5 of $7(71 \%)$, subdural collections in 1 of 7 (14\%), and venous distention sign ${ }^{5}$ in 5 of 7 (71\%). All patients, with the exception of 1 (patient 2), underwent patching with blood and/or fibrin glue (Tisseel; Baxter BioSurgery, Deerfield, Illinois) at our institution. Because we routinely measure opening pressure as part of the diagnostic evaluation of intracranial hypotension, pre-EBP opening pressures were available in all except this 1 patient. The average pre-EBP opening pressure was $8.6 \mathrm{~cm} \mathrm{H}_{2} \mathrm{O}$ (range, 1.0-15.0). A pre-EBP CT myelogram was available on all patients with $\mathrm{SIH}$ and showed active CSF leakage in 3 of 8 (38\%) patients. No patients in this case series underwent MR myelography with intrathecal gadolinium. The total volume of autologous blood and/or fibrin glue used for patching varied widely, with as little as $5 \mathrm{~mL}$ for 1 patient with a localized leak (patient 6), to as much as $42 \mathrm{~mL}$ (patient 9).

All patients had positional headache before blood patching. Pretreatment headache location was most commonly occipital $(n=6)$, with retro-orbital $(n=2)$ and holocephalic $(n=1)$ headaches less commonly present. Symptoms of intracranial hypotension had been present for an average of 12 months (range, 1 week to 2 years) before EBP.

\section{Characteristics after RIH Development}

The median time to repeat LP for confirmation of RIH after EBP was 1 day (range, 2 hours to 1 year). Six of 9 (66\%) patients had symptoms of RIH within 48 hours of blood patching. Headache location associated with RIH was different compared with the location of the pretreatment headache in 6 of $9(66 \%)$ patients, with the most common locations described being frontal (44\%) and peri- or retro-orbital (33\%). Eight of 9 (89\%) patients had new nausea and/or vomiting. New blurred vision was present in 7 of 9 (78\%) patients.

The average opening pressure among patients who had $\mathrm{RIH}$ symptoms was $30 \mathrm{~cm} \mathrm{H}_{2} \mathrm{O}$ (range, 22-55). Notably, 1 patient (patient 5) had an opening pressure of $>55 \mathrm{~cm} \mathrm{H}_{2} \mathrm{O}$ only 2 hours after blood patching, despite a pretreatment opening pressure of 1 $\mathrm{cm} \mathrm{H}_{2} \mathrm{O}$ and a patch volume of only $6 \mathrm{~mL}$.

All patients demonstrated symptomatic improvement after removal of CSF. In 8 of 9 cases, patients were started on oral acetazolamide and were treated for variable periods of time, ranging from 5 days to $3+$ years.

\section{DISCUSSION}

Although RIH is sparsely reported, in our experience it is not an uncommon complication of epidural blood patching. Although this case series was limited to patients with elevated opening pressure confirmed by lumbar puncture and therefore probably represents a subset of patients with a more severe spectrum of disease, we commonly encounter patients with similar, less severe symptoms after EBP. Although often self-limited and minor, RIH symptoms can at times be severe or persist well beyond the immediate posttreatment period. Lack of familiarity with this complication can result in misdiagnosis, including an incorrect attribution of symptoms to refractory intracranial hypotension.

The lack of more widespread recognition of this condition is probably caused by a superficial similarity of presenting features: headache is the predominant symptom experienced by patients with RIH and patients with intracranial hypotension. ${ }^{4}$ This case series highlights several typical features that are useful for discriminating persistent intracranial hypotension from $\mathrm{RIH}$.

First, patients who have RIH typically describe a change in headache phenotype. In contrast to the headaches of intracranial hypotension, which are commonly occipital in location, ${ }^{6}$ we found that headaches in RIH are most commonly frontal or peri-orbital in location (Fig 1). Additionally, headaches in intracranial hypotension are exacerbated with upright positioning, whereas those associated with RIH are typically worse when recumbent. Some patients report 


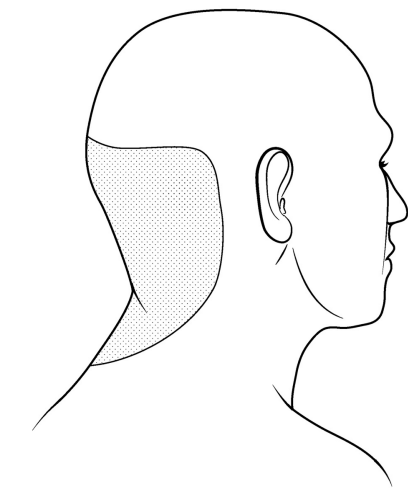

A

FIG 1. A, Patients presenting with intracranial hypotension most commonly - although not exclusively - complain of headaches in the occipital region. $B$, Patients who develop rebound intracranial hypertension often have a change in headache location, most commonly having headaches in a frontal, peri-orbital, or retro-orbital location. onset in that series ranged from 1 week to 9 months. Other authors have reported cases of RIH in which symptom onset was more rapid (ranging from immediate to 3 days). ${ }^{12-14}$ A single case report described a much more delayed onset of symptoms, occurring 2 years after blood patching. ${ }^{15}$ CSF pressure measurements in these series, when measured, ranged from 20-28 $\mathrm{cm} \mathrm{H}_{2} \mathrm{O}$. In general, the onset of symptoms in our series was more rapid, with a median time to onset of symptoms of 1 day, and the degree of CSF pressure elevation more severe, with an opening pressure of $>25 \mathrm{~cm} \mathrm{H}_{2} \mathrm{O}$ in $66 \%$ of patients. This may be caused in part by sampling bias; patients in our series only underwent lumbar puncture if symptoms were severe, whereas many patients clinically that the headaches associated with RIH are worse in the morning. These symptoms mirror features of those patients with raised intracranial pressure caused by other etiologies. ${ }^{7,8}$ Jugular venous outflow is impaired in the recumbent position, ${ }^{9}$ which probably accounts for the worsening of symptoms in some patients with RIH while lying down. Prolonged recumbent positioning at night may contribute to morning headaches, though other proposed mechanisms such as a rise in $\mathrm{pCO}_{2}$ at night, decreased CSF reabsorption, and increased CSF production modulated by chemical factors such as melatonin, for example, may also play a role. ${ }^{7,10}$ By contrast, headaches more prominent in the later part of the day are seen in some patients with intracranial hypotension. $^{11}$

Second, patients with acute RIH frequently have nausea, vomiting, and blurred vision, which are symptoms commonly found with elevated intracranial pressure. Whereas mild manifestations of these symptoms can occasionally be present at presentation in patients with intracranial hypotension, severe or continuous nausea, vomiting, and blurred vision are not common with low CSF pressure. The development of such symptoms immediately after blood patching should therefore raise suspicion for RIH.

Third, symptoms often develop in close proximity to the time of blood patching. Onset within 24-48 hours of treatment is common, and patients should be monitored most closely during this time period. In our series, onset even within several hours of EBP was not rare. In some cases, patients may even begin to have symptoms during the patching procedure itself while still on the procedure table. Nevertheless, not all patients in our series developed symptoms acutely, and a more delayed onset of symptoms should therefore not exclude the possibility of RIH. In our experience, patients who have development of symptoms over a more prolonged time period generally complain of less severe symptoms than those who have RIH more acutely, though the overall course of their symptoms may be more protracted.

Literature reports of RIH have been limited, with only 8 cases previously described, to our knowledge. The largest previous case series by Mokri ${ }^{4}$ reported 4 patients. Three of those patients had CSF pressure confirmed by lumbar puncture; the other patient was diagnosed by the presence of papilledema. Symptom suspected of having mild RIH were treated empirically without a confirmatory lumbar puncture. Thus, patients with milder CSF pressure elevations and/or more gradual symptom onset would not have been likely to be included in this investigation. We used $20 \mathrm{~cm} \mathrm{H}_{2} \mathrm{O}$ as the threshold for inclusion both because it replicates the criteria of prior reports of the condition and because it is a generally accepted upper limit of normal for CSF pressure in nonobese patients. ${ }^{16}$ It is possible, however, that the relative change in CSF pressure contributes to the development of symptoms independent of the absolute CSF pressure.

The mechanism behind the development of RIH is unknown. One potential explanation could be the immediate displacement of CSF from the enclosed confines of the spinal canal caused by the addition of patching material in the epidural space, resulting in increased pressure. Although this mechanism probably exists to some degree in all patients undergoing epidural patching, the degree of pressure increase caused by this mechanism would be expected to be proportional to the volume of patching material used; this was not always seen in our series. For example, 3 patients in our series had development of RIH despite the use of $<10$ $\mathrm{mL}$ patching material, and yet many other patients who receive EBP by use of volumes of $20-30 \mathrm{~mL}$ or more do not have RIH. An additional mechanistic possibility could be considered: physiologic mechanisms are engaged in patients with intracranial hypotension to compensate for decreased CSF volume, such as distention of venous structures in the head (as demonstrated by the "venous distention sign") or in the cervical spinal canal. ${ }^{5,17}$ When CSF volume is acutely returned to normal, failure of these compensatory mechanisms to immediately reverse could result in increased intracranial pressure. This explanation does not satisfactorily explain cases of delayed development of RIH, however. Mokri ${ }^{4}$ proposed several other possible mechanisms for RIH including upregulation of CSF production or disrupted CSF reabsorption in the setting of prolonged CSF leak, or return to a preexisting state of (previously unrecognized) idiopathic intracranial hypertension. All of these potential mechanisms, he concluded, were vulnerable to criticism for various reasons. Ultimately, it is possible that multiple different pathways exist that result in raised 
intracranial pressure and that variable engagement of 1 or more of these pathways may influence the time to onset and severity of symptoms among different patients.

Treatment of RIH in our series consisted of CSF drainage at the time of lumbar puncture, which immediately reduced, and in some cases eliminated, symptoms in all patients. Because the effect of CSF drainage is temporary, most patients were subsequently started on acetazolamide. Acetazolamide is an inhibitor of carbonic anhydrase that decreases CSF production through the inhibition of sodium-hydrogen ion exchange across the choroid plexus. ${ }^{18}$ We typically use an oral starting dose of $250 \mathrm{mg}$ twice per day and increase as necessary to control symptoms. Some patients may not be able to tolerate the side effects of acetazolamide, which include paresthesias of the digits, fatigue, and nausea. ${ }^{19}$ If patients cannot tolerate acetazolamide, we have used topiramate as an alternative because it also demonstrates carbonic anhydrase inhibitory effect and has been studied as an alternative to acetazolamide in cases of idiopathic intracranial hypertension. ${ }^{20,21}$ Acetazolamide should be used cautiously in patients with sulfa allergy, though our experience is that it is well tolerated by most patients with a history of reaction to other sulfa drugs, a finding that agrees with previous reports of the use of acetazolamide in sulfa-allergic patients. ${ }^{22}$ Other reported strategies for the treatment of RIH include the use of IV glycerol and the need for CSF shunting. ${ }^{1,13,14}$ Although we do not routinely admit patients overnight after their procedure, we do explicitly counsel patients on the symptoms that might suggest RIH and follow-up with them frequently by telephone after the procedure, in addition to providing them with emergency contact information should symptoms of RIH develop.

There are several limitations to this investigation. First, by only including patients with an opening pressure of $>20 \mathrm{~cm} \mathrm{H}_{2} \mathrm{O}$, we probably have excluded some patients with milder, but nonetheless symptomatic, elevations in CSF pressure. These patients may have a relatively increased CSF pressure compared with their pretreatment baseline but with opening pressures that still fall in the normal range. Second, because most patients who undergo epidural blood patching do not undergo routine subsequent CSF pressure evaluation, asymptomatic rises in CSF pressure in some patients could occur. Finally, and perhaps most importantly, we included only patients with symptoms severe enough to warrant repeat lumbar puncture. It is possible that RIH may present with different, less severe, clinical characteristics in patients with less dramatic elevations in CSF pressure.

\section{CONCLUSIONS}

$\mathrm{RIH}$ is a potential complication of epidural blood patching that should be considered in patients who report headache after treatment. Particularly suggestive features include change in headache phenotype, development of new nausea, vomiting, or blurred vision, and symptom development in close relationship to blood patching. Patients should be monitored closely for development of these symptoms, and can be treated with CSF drainage and/or oral acetazolamide.

\section{REFERENCES}

1. Mokri B. Spontaneous low pressure, low CSF volume headaches: spontaneous CSF leaks. Headache 2013;53:1034-53

2. Sencakova D, Mokri B, McClelland RL. The efficacy of epidural blood patch in spontaneous CSF leaks. Neurology 2001;57:1921-23

3. Berroir S, Loisel B, Ducros A, et al. Early epidural blood patch in spontaneous intracranial hypotension. Neurology 2004;63:1950-51

4. Mokri B. Intracranial hypertension after treatment of spontaneous cerebrospinal fluid leaks. Mayo Clinic Proc 2002;77:1241-46

5. Farb RI, Forghani R, Lee SK, et al. The venous distension sign: a diagnostic sign of intracranial hypotension at MR imaging of the brain. AJNR Am J Neuroradiol 2007;28:1489-93

6. Schievink WI. Spontaneous spinal cerebrospinal fluid leaks and intracranial hypotension. JAMA 2006;295:2286-96

7. Dunn LT. Raised intracranial pressure. J Neurol Neurosurg Psychiatry 2002;73(Suppl 1):i23-27

8. Sergott RC. Headaches associated with papilledema. Curr Pain Headache Rep 2012;16:354-58

9. Valdueza JM, von Munster T, Hoffman O, et al. Postural dependency of the cerebral venous outflow. Lancet 2000;355:200-01

10. Nilsson C, Ståhlberg F, Gideon P, et al. The nocturnal increase in human cerebrospinal fluid production is inhibited by a beta 1-receptor antagonist. Am J Physiol 1994;267:R1445-48

11. Leep Hunderfund AN, Mokri B. Second-half-of-the-day headache as a manifestation of spontaneous CSF leak. J Neurol 2012;259: 306-10

12. Cestari DM, Rizzo JF. Intracranial hypertension following epidural blood patch. Neurology 2003;61:1303

13. Lin W-C, Lee M-H, Kao Y-F, et al. Monitoring rebound intracranial hypertension by cine MRI after treatment of spontaneous intracranial hypotension in a patient with notable subdural hemorrhage. Eur J Radiol Extra 2009;69:e1-e4

14. Tsui H, Wu S, Kuo H, et al. Rebound intracranial hypertension after treatment of spontaneous intracranial hypotension. Eur J Neurol 2006;13:780-82

15. Philipps J, Busse O. From low to high: late-onset intracranial hypertension after treatment of spontaneous intracranial hypotension. J Neurol 2007;254:956-57

16. Whiteley $\mathrm{W}$, Al-Shahi R, Warlow $\mathrm{CP}$, et al. CSF opening pressure: reference interval and the effect of body mass index. Neurology 2006;67:1690-91

17. Medina JH, Abrams K, Falcone S, et al. Spinal imaging findings in spontaneous intracranial hypotension. Am J Roentgenol 2010;195: 459-64

18. Johanson CE, Murphy VA. Acetazolamide and insulin alter choroid plexus epithelial cell $[\mathrm{Na}+], \mathbf{p H}$, and volume. Am J Physiol 1990; 258:F1538-46

19. Ball AK, Clarke CE. Idiopathic intracranial hypertension. Lancet Neurol 2006;5:433-42

20. Celebisoy N, Gökçay F, Sirin H, et al. Treatment of idiopathic intracranial hypertension: topiramate vs acetazolamide, an open-label study. Acta Neurol Scand 2007;116:322-27

21. Shah VA, Fung S, Shahbaz R, et al. Idiopathic intracranial hypertension. Ophthalmology 2007;114:617.e611-617.e612

22. Lee AG, Anderson R, Kardon RH, et al. Presumed "sulfa allergy" in patients with intracranial hypertension treated with acetazolamide or furosemide: cross-reactivity, myth or reality? Am J Ophthalmol 2004;138:114-18 